\title{
International Journal of
}

\section{Computer Network and Information Security}

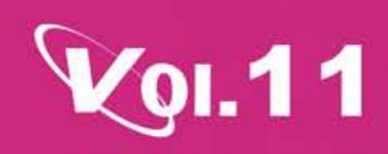

No.12 Dec. 2019

\section{IJCNIS KoI.11}

Http:// www.mecs-press.org

Vol. 11 No.12 December 2019
Modern Education

and Computer Science PRESS 
International Journal of Computer Network and Information Security (IJCNIS)

ISSN Print: 2074-9090, ISSN Online: 2074-9104

Volume 11, Number 12, December 2019

\section{Contents}

\section{REGULAR PAPERS}

Performance Analysis of Network Applications on IPv6 Cloud Connected Virtual Machine

Zeeshan Ashraf, Adnan Sohail, Muhammad Yousaf

Design and Implementation of Runtime Reconfigurable Encryption Algorithms using Custom ICAP 10

Processor

Jamuna S, Dinesha P, K PShashikala, Kishore Kumar K

IT Risk Management Based on ISO 31000 and OWASP Framework using OSINT at the Information

Gathering Stage (Case Study: X Company)

Anak Agung Bagus Arya Wiradarma, Gusti Made Arya Sasmita

Rita Ganguly, Anirban Sarkar

Odukoya Oluwatoyin, Akinyemi Bodunde, Gooding Titus, Aderounmu Ganiyu 